\title{
Non-Healing Hematomas and Easy Bruising: A Case Report on the Ehlers-Danlos Syndrome of the Hypermobility Type
}

\section{Da Wang and Marinus CG van Praag* \\ Department of dermatology, Franciscus Gasthuis, The Netherlands}

*Corresponding author: Marinus CG van Praag, Franciscus Gasthuis, Kleiweg 500, 3045 PM, Rotterdam, South-Holland, The Netherlands, Tel: +3110 461 6131; Email: m.vanpraag@franciscus.nl

\section{Case Report \\ Volume 4 Issue 3}

Received Date: August 05, 2019

Published Date: September 04, 2019

DOI: $10.23880 /$ cdoaj- 16000188

\section{Abstract}

Introduction: Ehlers-Danlos syndrome (EDS) of the hypermobility type is characterized by hyperextensibility of the skin, hypermobility of joints and skin fragility leading to easy bruising and delayed wound healing with atrophic scarring. Unfortunately, EDS remains often unrecognized.

Case Report: We report a 54-year old Caucasian woman who presented at our clinic with bruises on both legs without any trauma in advance. Careful anamnesis revealed a tendency to bruising, delayed wound healing, hypermobility of the joints, myalgia and arthralgia. As a child she often dislocated her joints after which she was able to reposition them herself. She had a slow motoric development. In the past, she had multiple rib contusions and a rib fracture after minor physical activities. Physical examination showed a normal posture. The skin was soft and mild stretchable with stretch marks and hypertrophic scarring in certain areas of the body. On both upper legs there are signs of hematomas. She has a Beighton score of 6/9. After reviewing the guidelines of diagnosing EDS of the hypermobility type, she met all the criteria. DNA tests for other types of EDS and connective tissue related diseases were negative. We referred her to the rehabilitation specialist for guidance.

Conclusion: A huge delay in the diagnosis of EDS of the hypermobility type is often seen. By reporting this case, we would like to raise awareness for this disease in order to shorten the delay in diagnosis and in consequence offering patients the adequate treatment.

Keywords: Ehlers-danlos syndrome; Hypermobilitiy

\section{Introduction}

The Ehlers-Danlos syndrome (EDS) is a rare inherited heterogeneous group of disorders that affect the connective tissue supporting the skin, blood vessels, bones and organs. It is characterized by hyperextensibility of the skin, hypermobility of joints and skin fragility leading to easy bruising and delayed wound healing with 
atrophic scarring. The disease is named after Edvard Lauritz Ehlers (1863-1937), a Danish dermatologist, and Henri-Alexandre Danlos (1844-1912), a French physician, who each published cases of patients with joint laxity, hyperextensible skin, a tendency to bruising and skin lesions [1]. There are many subtypes of this disease. The 1988 'Berlin Nosology' reported 11 subtypes characterized by Roman numerals based on clinical findings and the fashion of inheritance [2]. In 1998, a revised classification, the 'Villefranche Nosology', was published in which 6 subtypes were described according to clinical signs rather than the method of inheritance [3]. With the advancement of biotechnology, new subtypes were able to be identified. The most recent classification was published in 2017 and recognizes 13 subtypes of which the hypermobility and classical forms are most common [4]. Worldwide, at least 1:5000 individuals are estimated to be affected with EDS in general [5]. Unfortunately, EDS usually remains unrecognized at first visit as most patients present with vague complaints without any significant leads after conducting patient history and routine investigation. Furthermore, due to the more specialized medical care that hospitals provide, EDS is easily overlooked as it comprises many medical disciplines. As a consequence, there is often a delay in diagnosing these patients unable to offer the adequate management which may lead to severe disability. Our case report illustrates a patient with EDS of the hypermobility type presenting with hematomas of the legs. By demonstrating this case, we would like to raise awareness for this disease in order to shorten the delay in diagnosis.

\section{Case Report}

A 54-year old Caucasian woman presented at our practice with bruises on both legs since 4 days with a burning sensation. There was no trauma prior to these bruises. Furthermore, she mentioned that she has a tendency to bruising but the burning sensation was novel. She is able to predict the development of these bruises as there is a period of 'not feeling well' leading up to these skin lesions. Besides her skin lesions, she has a history of rheumatoid arthritis, scoliosis, asthma, diaphragmatic hernia, Tietze syndrome, recurring urine tract infections, sleep apnea and Scheuermann's disease. She just quit smoking cigarettes 1 week prior to the hospital visit with a smoking history of 30 years. Physical examination revealed on both upper legs ventrally multiple blue colored maculas with erythema of nummular to a child's hand palm size (Figures $1 \& 2$ ). The site of an old skin biopsy, that she got years ago, did not heal well and left a hypertrophic scar behind (Figure 3). Tests were conducted on different clotting factors and ruled out any bleeding disorders. We looked for potential syndromes that could fit her easy bruising and non-healing hematomas, along with potentially her other co-existing diseases. Easy bruising and non-healing hematomas are symptoms of EDS. Therefore, we followed with an EDS focused patient history and physical examination in cooperation with a clinical geneticist.

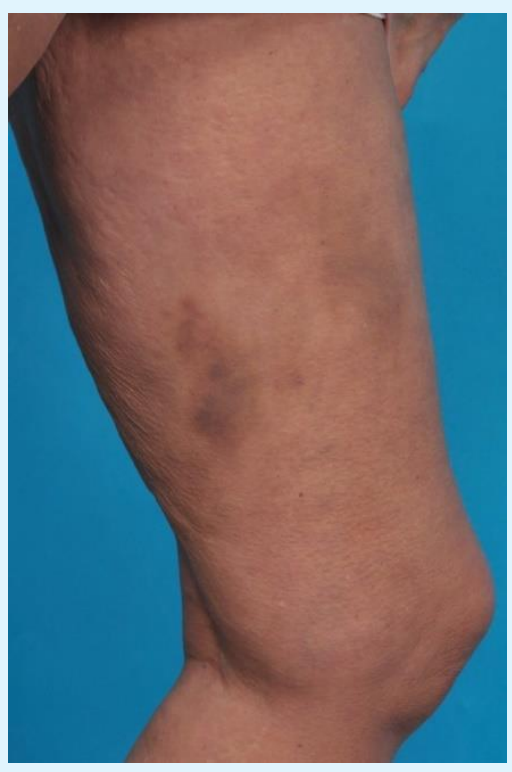

Figure 1: The bruises on her upper left leg.

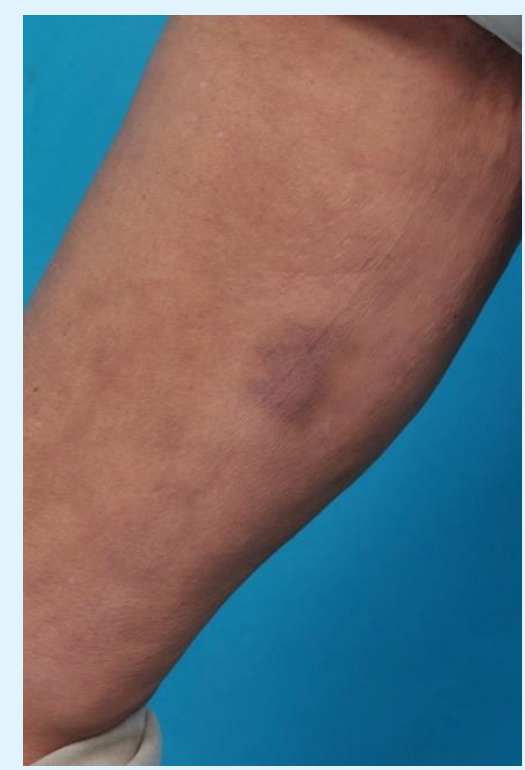

Figure 2: The bruises on her upper right leg. 


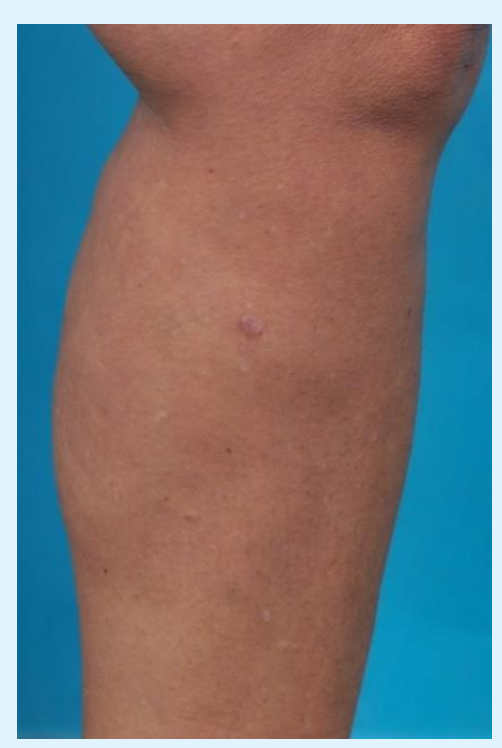

Figure 3: Hypertrofic scarring at the biopsy site (lower left leg), taken 1.5 years ago.

It appears that she as a child was hypermobile. She could put her legs behind her head and often encountered joint luxation which she was able to reposition herself. She is still able to hyperextend her thumb (Figure 4).

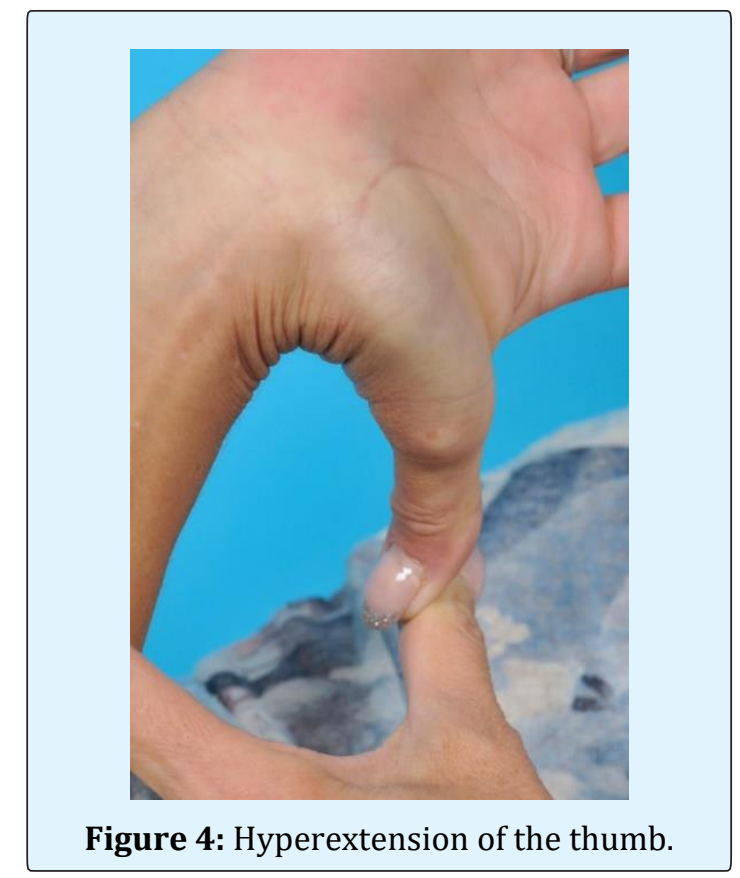

All her life she suffered from pain throughout her whole body and joint pain. She often sprains her ankle as well. Also, she struggles with wound healing. Stitching was not possible due to a thin skin according to her. Patient has multiple stretch marks but no varices. During childhood, she had a slow motoric development. She never crawled. Balancing and coordination were always a problem for her. In the digestive area, she has reflux and irritable bowel syndrome. Furthermore, she has recurring stomatitis aphtosa in her mouth. Besides, she has myopia $(-2.75$ both eyes). In the past few years she encountered multiple rib contusions after doing minor physical activities and a rib fracture after rolling out a yoga mat. A few years ago, she was examined by a cardiologist due to a high systolic blood pressure $(>220 \mathrm{mmHg}$ ) that was supposedly based on a non-functioning thyroid. Also, she suffered from complaints such as a hanging eye and mouth in the past. She also mentioned a bad short-term memory.

Family history revealed that she is a child of non-blood related parents and has 6 siblings. Her mother died at age 79 and had suffered from a double lung embolism and kidney cysts. Her father had a heart attack below the age of 50 and suffered from Scheuermann's disease. Her uncle had died suddenly during work. Heart- and vascular disease was often occurring on the father's side of the family. Her oldest brother suffered from a heart attack at age 45 and received an ICD implant at age 57 due to arrhythmias. The second eldest brother also has Scheuermann's disease and Tietze's syndrome and had undergone an operation for most likely diaphragmatic hernia. Her youngest brother is also very flexible, has joint issues and vitiligo.

Physical examination revealed a height of $160,5 \mathrm{~cm}$ and a weight of $65 \mathrm{~kg}$. There is a normal posture without signs of a Marfan habitus. Her palate was normal with a small uvula. There was no blue sclera. Inspection of the skin showed mild stretchable and soft skin. The abdominal area showed petechiae. Furthermore, there were stretch marks on her lower back, thighs and upper legs. She had, except for the old biopsy scar, scars located on her head and back. The thoracic area had no signs of pectus carinatum or excavatum but showed a mild scoliosis and kyphosis. Her extremities did not show signs of poly-/syn-/clyno-/aranchnodactyly. She had a pes planus on the left side and a sagged foot on the right side. There were no pretibial abnormalities or herniation of fat on the lateral side of the feet. Beighton score: 6/9.

In our case, the patient suffers from EDS of the hypermobility type. She underwent DNA tests to exclude other types of EDS, including the vascular type, and other soft tissue related diseases. While waiting for these 


\section{Clinical Dermatology Open Access Journal}

results, we referred her to vascular medicine for a checkup because of her positive family history for heart- and vascular diseases. Fortunately, there were no vascular abnormalities. The DNA tests came in negative. A referral was written to the rehabilitation specialist for guidance in daily activities and coping with physical complaints. Advice was given to reduce intensive activities in order to diminish pain. Muscle strengthening exercises were favorable without burdening the joints extensively.

\section{Discussion}

EDS of the hypermobility type is typically characterized by general joint hypermobility, arthralgia, myalgia, soft tissue injuries and arthritis [6]. It is an autosomal dominant disease which means that there is $50 \%$ chance of inheritance by the offspring [7]. Pain is the main complaint of patients of EDS of the hypermobility type. These symptoms are in line of those of our patient. Since EDS of the hypermobility type has no known diagnostic molecular defects yet, no laboratory diagnostic test can be done. Hence, EDS of the hypermobile type is a clinical diagnosis. According to the new classification of the International EDS Consortium, there are 3 criteria that must be met in order to diagnose the EDS of the hypermobility type (Table 1) [7]. Herein, the Beighton score and the Brighton criteria play a key role in diagnosing generalized joint hypermobility (Tables 2\&3).
The Beighton score is a part of the Brighton criteria, which consists of major and minor criteria in diagnosing general joint hypermobility (Tables 2\&3) [6,7]. This is diagnosed in the presence of 2 major criteria, or 1 major and 2 minor criteria, or 4 minor criteria. Two minor criteria will be sufficient in case of an effected first-degree relative. Our patient met all 3 criteria for diagnosing EDS of the hypermobility type. Concerning the Brighton criteria, our patient met the 2 major criteria with a Beighton score of 6/9. EDS is a multi-systemic disorder (Table 4) [6]. In retrospect, our patient showed multiple symptoms of EDS of the hypermobility type that could have led to the diagnosis.

Treatment of EDS of the hypermobility type is mainly coping with pain. Pain should be managed in which it is acceptable rather than pain free [6,7]. Herein, the physiotherapist or the rehabilitation specialist plays an important role. Besides, patients should be referred to a cardiologist/vascular specialist, orthopedic surgeon, gastro-enterologist, ophthalmologist, urologist and neurologist when related symptoms occur. When encountering surgeries, careful suturing and sutures staying longer in place should be considered. Local anesthetic resistance has been reported and should be considered. Ascorbic acid (2 g/d for adults) may reduce bruising. Deamino-Delta-D-Arginine Vasopressin (DDAVP) may be useful to normalize bleeding time.

\section{Criteria}

1. Generalized joint hypermobility (a cut-off Beighton score $\geq 6$ for prepubertal children and adolescents, $\geq 5$ for pubertal men and women up to the age of 50 , and $\geq 4$ for those $>50$ years of age), minor adaptations and assessment of alternative joints are also suggested in certain situations (eg, age, acquired joint imitations due to injury/surgery etc).

2. Two of more of the following features: (a) systemic manifestations of generalized connective tissue disorder, (b) positive family history, (c) Musculoskeletal complications (chronic pain $>3$ months and/or recurrent joint dislocations/instability).

3. Absence of unusual skin fragility as of other types of EDS and exclusion of alternative heritable/acquired connective tissue disorder including autoimmune rheumatologic conditions.

Table 1: Criteria in diagnosing EDS of the hypermobility type.

\begin{tabular}{|l|}
\hline \multicolumn{1}{|c|}{ Beighton score } \\
\hline Calculation: \\
\hline - 1 point if while standing forward bending you can place palms on the ground with legs straight \\
\hline - 1 point for each elbow that bends backwards \\
\hline - 1 point for each knee that bends backwards \\
\hline - 1 point for each thumb that touches the forearm when bent backwards \\
\hline - 1 point for each little finger that bends backwards beyond 90 degrees \\
\hline
\end{tabular}

Table 2: Beighton score. 


\section{Major criteria:}

\section{Brighton criteria}

- A Beighton score of $4 / 9$ or greater (either currently or historically)

- Arthralgia for longer than 3 months in 4 or more joints

Minor criteria:

- A Beighton score of 1,2 , or $3 / 9(0,1,2$, or 3 if aged $50+)$

- Arthralgia ( $>3$ months) in 1 or 3 joints or back pain ( $>3$ months), spondylosis/spondylolisthesis

- Dislocation/subluxation in $>1$ joint, or in 1 joint on more than 1 occasion

- Soft tissue rheumatism, $>3$ lesions (e.g. epicondylitis, tenosynovitis, bursitis)

- Marfanoid habitus (tall, slim, span:height ratio $>1.03$, upper:lower segment ratio less than 0.89 , arachnodactyly (positive Steinberg/wrist signs)

- Abnormal skin: striae, hyperextensibility, thin skin, papyraceous scarring

- Eye sings: drooping eyelids or myopia or antimongoloid slant

- Varicose veins or hernia or uterine/rectal proplaps

Table 3: Brighton criteria.

\begin{tabular}{|l|l|}
\hline \multicolumn{1}{|c|}{ System } & \multicolumn{1}{c|}{ Manifestation } \\
\hline Cardiovascular & $\begin{array}{l}\text { Aortic regurgitation, aortic root dilatation, mitral valve prolapse, mitral regurgitation, } \\
\text { tricuspid regurgutation, Reynaud phenomenon. }\end{array}$ \\
\hline $\begin{array}{l}\text { Autonomic Nervous } \\
\text { System }\end{array}$ & Palpitations, dizziness, pre-syncope, syncope. \\
\hline Gastrointestinal & $\begin{array}{l}\text { Gastroesophageal reflux, dyspepsia, gastritis, delayed gastric emptying, irritable bowel } \\
\text { syndrome. }\end{array}$ \\
\hline Hematologic & $\begin{array}{l}\text { Easy bruising, bleeding tendancy, prolonged bleeding time, oral mucosal bruises, } \\
\text { menometrorraghia. }\end{array}$ \\
\hline Ocular & Myopia, strabismus.* \\
\hline Gynecologic & Dysmenorrhea, menorrhagia, dyspareunia, uterine prolaps. \\
\hline Urologic & $\begin{array}{l}\text { Constipation, fecal soiling, urinary tract infections, urinary incontinence, bladder prolapse, } \\
\text { rectal prolapse. }\end{array}$ \\
\hline Neurologic & $\begin{array}{l}\text { Short labor and delivery, premature rupture of membranes, pelvic pain, varicose veins, } \\
\text { worsening of dysautonomia during pregnancy, postpartum hemorrhage, complicated } \\
\text { perineal wounds. }\end{array}$ \\
\hline Psychiatric & $\begin{array}{l}\text { Headache, local anesthesia failure, postural instability, increased frequency of falls, impaired } \\
\text { proprioceptive acuity, Chiari 1 type } 1 \text {. }^{*}\end{array}$ \\
\hline
\end{tabular}

Table 4: EDS is a multisystemic disorder.

* Patient's symptoms are indicated in red.

\section{Conclusion}

We report a case of EDS of the hypermobility type with a tendency of bruising, delayed healing of hematomas, hypermobility of the joints, arthralgia and myalgia. Due to the various medical areas that this disease can affect, patients often have a long history of hospital visits with different medical specialist prior the diagnosis. By reporting this case, we hope to alert other medical care takers to look beyond their own discipline when encountering patients with similar symptoms. Future research should be addressed on defining its genetic aberrations in order to facilitate the diagnosis.

\section{References}

1. Parapia LA, Jackson C (2008) Ehlers-Danlos syndrome - a historical review. Br J Haematol 141(1): 32-35. 
2. Beighton P, de Paepe A, Danks D, Finidori G, GeddeDahl T, et al. (1986) International nosology of heritable disorders of connective tissue, Berlin. Am J Med Genet 29(3): 581-594.

3. Beighton P, De Paepe A, Steinmann B, Tsipouras P, Wenstrup RJ (1997) Ehlers-Danlos syndromes: Revised nosology, villefranche, ehlers-Danlos national foundation (USA) and ehlers-Danlos support group (UK). Am J Med Genet 77(1): 31-37.

4. Malfait F, Francomano C, Byers P, Belmont J, Berglund B, et al. (2017) The 2017 International Classification of the Ehlers-Danlos Syndromes. Am J Med Genet C Semin Med Genet 175(1): 8-26.
5. Ehlers-Danlos syndrome. USA.gov.

6. Yael G, Giris J, Grahame R (2016) Ehlers-Danlos Syndrome - hypermobility type: a much neglected multisystemic disorder. Rambam Maimonides Med J $7(4)$.

7. Zhang W, Windsor K, Jones R, Taunton DO (2019) Hypermobile type Ehlers-Danlos syndrome associated with hypogammaglobulinemia and fibromyalgia: A case-based review on new classification, diagnosis, and multidisciplinary management. Clin Case Rep 7(4): 680-685. 